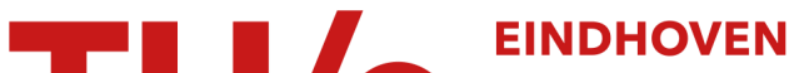 UNIVERSITY OF TECHNOLOGY
}

\section{Optical bandwidth and fabrication tolerances of multimode interference couplers}

\section{Citation for published version (APA):}

Besse, P. A., Bachmann, M., Melchior, H., Soldano, L. B., \& Smit, M. K. (1994). Optical bandwidth and fabrication tolerances of multimode interference couplers. Journal of Lightwave Technology, 12(6), 1004-1009.

\section{Document status and date:}

Published: 01/01/1994

\section{Document Version:}

Publisher's PDF, also known as Version of Record (includes final page, issue and volume numbers)

\section{Please check the document version of this publication:}

- A submitted manuscript is the version of the article upon submission and before peer-review. There can be important differences between the submitted version and the official published version of record. People interested in the research are advised to contact the author for the final version of the publication, or visit the $\mathrm{DOI}$ to the publisher's website.

- The final author version and the galley proof are versions of the publication after peer review.

- The final published version features the final layout of the paper including the volume, issue and page numbers.

Link to publication

\section{General rights}

Copyright and moral rights for the publications made accessible in the public portal are retained by the authors and/or other copyright owners and it is a condition of accessing publications that users recognise and abide by the legal requirements associated with these rights.

- Users may download and print one copy of any publication from the public portal for the purpose of private study or research.

- You may not further distribute the material or use it for any profit-making activity or commercial gain

- You may freely distribute the URL identifying the publication in the public portal.

If the publication is distributed under the terms of Article 25fa of the Dutch Copyright Act, indicated by the "Taverne" license above, please follow below link for the End User Agreement:

www.tue.nl/taverne

Take down policy

If you believe that this document breaches copyright please contact us at:

openaccess@tue.nl

providing details and we will investigate your claim. 


\title{
Optical Bandwidth and Fabrication Tolerances of Multimode Interference Couplers
}

\author{
Pierre A. Besse, Maurus Bachmann, H. Melchior, L. B. Soldano, and M. K. Smit
}

\begin{abstract}
Analytical expressions are derived which relate the optical bandwidth and the fabrication tolerances of multimode interference (MMI) couplers to their most important design parameters. These expressions compare adequately with mode analysis simulations. For strong guided structures, the optical bandwidth is shown to be inversely proportional to the number of input and output ports $N$ and to the length of the device. The fabrication tolerances are independent of $N$ and proportional to the output channel separation $D$. Measurements on MMI couplers in InP/InGaAsP corroborate the theoretical predictions.
\end{abstract}

\section{INTRODUCTION}

M ULTIMODE interference (MMI) couplers, based on the self-imaging effect [1], [2], are rapidly gaining popularity. MMI couplers have already been applied as power splitters and combiners [3], [4], in polarization-independent switches [5], [6], in phase-diversity optical receivers [7], [8], and in ring semiconductor lasers [9]. The main advantages of such devices are low-loss, ultracompact size [10], and large fabrication tolerances. Furthermore, polarization-independent components can be realized by using strongly guided waveguide structures [5].

As future WDM communication networks use erbiumdoped fiber amplifiers with bandwidths of the gain spectra of $20 \mathrm{~nm}$ and more, integrated components will need at least such large optical bandwidths. The aim of this paper is therefore to study the bandwidth capabilities of MMI couplers and to quantify their fabrication-related tolerances in performance.

First, the dependency of these properties on the geometrical design parameters, as well as on the number of input and output ports is discussed. We distinguish between different kinds of MMI couplers [11] as shown in Fig. 1. Depending on the design, $N \times N, 1 \times N$, or $2 \times N$, self-imaging properties are obtained. For all these different cases, simple analytical expressions are derived using a Gaussian beam approximation. We find that the optical bandwidth is inversely proportional to the number of ports and to the length of the device. The fabrication tolerances remain independent of $N$ and are proportional to the output channel separation $D$.

Manuscript received November 3, 1993. This work was supported in part by the RACE II ATMOS Project, by the Swiss Federal Office for Education and Science, and by the Technical Sciences Foundation (STW) within the program of the Dutch Foundation for Fundamental Research on Matter (FOM).

P. A. Besse, M. Bachmann, and H. Melchior are with the Micro- and Optoelectronics Group, HPT F10, Institute of Quantum Electronics, ETH Zurich, CH-8093 Zurich, Switzerland.

L. B. Soldano and M. K. Smit are with the Integrated Optics Group, Technical University of Delft, Delft, The Netherlands.

IEEE Log Number 9401727

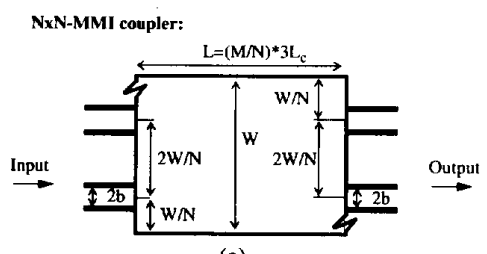

(a)

IxN MMI-coupler:

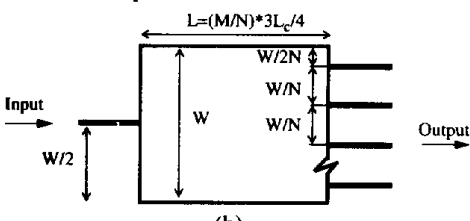

(b)

2xN MMI-coupler:

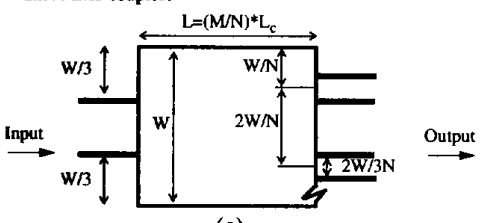

(c)

Fig. 1. Geometrical design of multimode interference (MMI) couplers of length $L=(M / N) \cdot 3 L_{c} / a$. (a) $N \times N$ self-images using complete interference, $a=1, b=$ free parameter. (b) Symmetrical $1 \times N$ self-images. $a=4$. Input at $W / 2$. (c) $2 \times N$ self-images using restricted interference. $a=3$. Input at $W / 3$ or $2 W / 3$.

In the second part of the paper, we present optical bandwidth measurements we obtained on $2 \times 2$ restricted interference MMI couplers used as $3 \mathrm{~dB}$ power splitters [12] and as cross couplers. In these restricted interference devices, the access waveguides are positioned in order not to excite the modes $2,5,8, \cdots$ of the multimode section; this permits the realization of short couplers [10]. The analytical approximations compare well with the experimental results, and also with the predictions of the more sophisticated mode analysis (MA) technique [12].

\section{THEORY}

We first consider the MMI coupler of Fig. 2. The behavior of the device can be described by using the paraxial approximation in strong guided structures [10]. The refractive index profile in the $y$ direction is replaced by the effective index $n$, so that only a two-dimensional problem has to be solved.

\section{A. Analytical Approximation}

Depending on the ratio of the MMI coupler length $L$ to the square of the MMI coupler width $W$, and on the lateral 


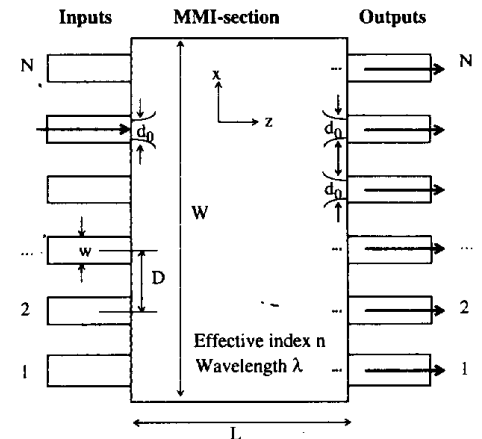

Fig. 2. Multimode interference (MMI) coupler with $N$ inputs and $N$ outputs. The inputs and their $N$ self-images at the outputs are approximated by Gaussian beams with waist $d_{0}$.

positions of the input and output waveguides, different selfimaging arrangements are obtained [10]. As the self-imaging depends on the interference of the different eigenmodes, the coupling length $L_{c}$ between the first two lowest order eigenmodes of the MMI coupler is used as a characteristic dimension:

$$
L_{c} \equiv \frac{\pi}{\left(\beta_{0}-\beta_{1}\right)} \equiv \frac{4}{3} n \frac{W_{\mathrm{eq}}^{2}}{\lambda}
$$

with $\beta_{0}$ and $\beta_{1}$ corresponding to the propagation constants of the first two modes. The last part of the equation can be considered as a definition of the equivalent width $W_{\text {eq }}$ of the MMI coupler. For strongly guiding structure, we get $W_{\text {eq }} \cong W$. Self-images of equal intensities appear in an MMI coupler, whose length $L$ is given by

$$
L=\frac{M}{N} \cdot \frac{3 L_{c}}{a} \cong \frac{M}{N} \cdot \frac{4 n}{a} \cdot \frac{W^{2}}{\lambda}
$$

where $N$ is the number of images. $M$ is an integer without a common divisor with $N$ that defines the several possible device lengths where $N$ self-images appeared. The effective index of the central slab section of the MMI coupler is represented by $n$. The integer $a$ characterizes the kind of MMI coupler, as explained in Fig. 1. The general $N \times N$ MMI coupler has $a=1$, and the lateral positions of the inputs contain a free parameter $b[10]$. For a symmetrical $1 \times N$ MMI coupler [3], the input is at $W / 2$ and $a=4$. The $2 \times N$ restricted interference MMI coupler has $a=3$, and the inputs should be located at $W / 3$ and $2 W / 3$ [13]. The relative phase relations at the outputs of strongly guiding MMI couplers can be analytically expressed [11] using paraxial approximation.

Once the length of the MMI coupler is given, the wavelength $\lambda_{\text {opt }}$ for the optimal self-imaging process is expressed from (2) by

$$
\lambda_{\mathrm{opt}}=\frac{M}{N} \cdot \frac{4}{a L} \cdot\left(n \cdot W^{2}\right) .
$$

The term in parentheses is polarization dependent and, strictly speaking, we would have to define two optimal wavelengths $\lambda_{\text {opt }}^{\mathrm{TE}}$ and $\lambda_{\text {opt }}^{\mathrm{TM}}$ for TE and TM, respectively. For strong guided structures, the wavelength dependency almost disappears.
From (2) the variations of the wavelength, the width, and the length of the MMI coupler are related by

$$
\frac{|\partial \lambda|}{\lambda_{\mathrm{opt}}}=2 \frac{|\partial W|}{W}=\frac{|\partial L|}{L} .
$$

For well-separated output images, these variations lead first to an increase of the losses, the splitting ratio remaining more or less constant [12]. We therefore describe the optical bandwidth $2|\partial \lambda|$ and the fabrication tolerances $\partial W$ in terms of excess losses. As shown in Fig. 4(a), the optical bandwidth $2|\partial \lambda|$ gives the whole wavelength range centered around $\lambda_{\text {opt }}$ that leads to an excess loss lower than a value $\alpha$. We choose to express the fabrication tolerances by the width variation since this geometrical parameter is the most critical one in strongly guided MMI couplers. The fabrication tolerances $\partial W$ express only the half of the possible range of width variations to remain under the excess loss $\alpha$ [Fig. 4(b)].

In order to get close expressions for $2|\partial \lambda|$ and $\partial W$, we calculate the excess losses $\alpha$ as a function of the MMI length variation $|\partial L|$ and introduce the result in (4). The function $\alpha(\delta L)$ can directly be expressed by overlapping the optical field amplitudes at the end of the MMI section with the fields of the output waveguides. Since we consider well-separated output images, this calculation has to be performed at only one output. The optical field amplitudes of the monomode symmetrical input and output waveguides can be approximated by Gaussian beams of waist $d_{0}$. By calculating the overlap $T$ of such a Gaussian beam focused at $L$ with the same Gaussian beam but focused at $L+\delta L$, the length variation $\delta L$ can be related to the excess losses $\alpha \equiv-10 \cdot \log _{10}(T)$ as follows (Appendix):

$$
\begin{gathered}
|\delta L| \leq Z \cdot d_{0}^{2} \cdot \frac{\pi n}{2 \lambda_{\mathrm{opt}}} \text { with } \\
Z=\left[\frac{\left(4-5 T^{2}\right)+\sqrt{\left(4-5 T^{2}\right)^{2}-16 T^{2}\left(T^{2}-1\right)}}{8 T^{2}}\right]^{1 / 2} .
\end{gathered}
$$

Introducing this result in (4), we get for the optical bandwidth $2|\partial \lambda|$ and for the fabrication tolerances $\partial W$,

$$
\begin{gathered}
2|\delta \lambda| \cong Z(\alpha) \cdot \frac{\pi}{4} \cdot \frac{a N}{M} \cdot \frac{d_{0}^{2}}{W^{2}} \cdot \lambda_{\text {opt }} \quad \text { and } \\
|\delta W| \leq Z(\alpha) \cdot \frac{\pi}{16} \cdot \frac{a N}{M} \cdot \frac{d_{0}^{2}}{W} .
\end{gathered}
$$

These expressions apply for a general design of the MMI couplers. In most of the practical applications, the special design of MMI couplers with equidistant inputs (or outputs) is used. In this important case, the width $W$ can be written as $W \cong N \cdot D$ with $D$ the spacing of the channels measured between the axes of the waveguide. Equation (6) then leads to

$$
\begin{gathered}
2|\delta \lambda| \cong Z(\alpha) \cdot \frac{\pi}{4} \cdot \frac{a}{N M} \cdot\left(\frac{d_{0}}{D}\right)^{2} \cdot \lambda_{\text {opt }} \quad \text { and } \\
|\delta W| \leq Z(\alpha) \cdot \frac{\pi}{16} \cdot \frac{a}{M} \cdot\left(\frac{d_{0}}{D}\right)^{2} \cdot D .
\end{gathered}
$$




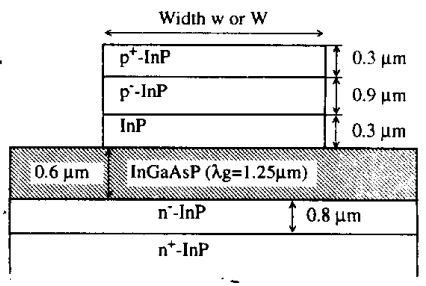

Fig. 3. Cross section of $\operatorname{InP} / \mathrm{nGaAsP}$ waveguide. The input and output waveguides have a width $w$, the MMI section has a width $W$. The quaternary material has a gap wavelength of $\lambda_{g}=1.25 \mu \mathrm{m}$.

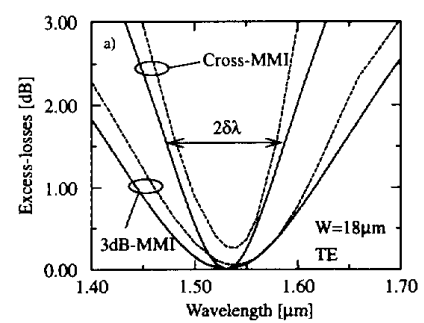

(a)

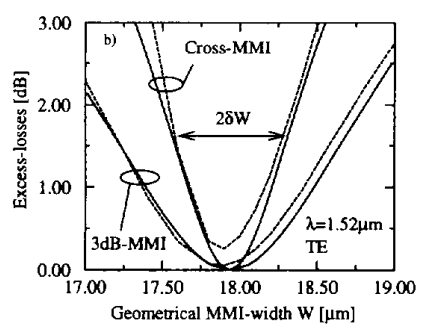

(b)

Fig. 4. (a) Optical bandwidth of $3 \mathrm{~dB}$ and cross-MMI couplers with restricted interference. Solid lines are calculated with (6). Dashed lines are the results of the MA. (b) Fabrication tolerances of $3 \mathrm{~dB}$ and cross-MMI couplers with restricted interference. Solid lines are calculated with (6). Dashed lines are the results of the MA. For $3 \mathrm{~dB}$ couplers, $a=3, N=2, M=1, L=530$ $\mu \mathrm{m}, d_{0}=3.4 \mu \mathrm{m}, n=3.3$, TE polarization. For cross couplers, $a=3$, $N=1, M=1, L=1060 \mu \mathrm{m}, d_{0}=3.4 \mu \mathrm{m}, n=3.3$, TE polarization.

Equations (6) and (7) have been written as a function of fixed design parameters, namely, of the "acceptable" excess losses $\alpha$, of the kind of MMI coupler used $(a, N, M)$, of the geometrical parameters at the inputs/outputs $\left(d_{0}, W, D\right)$, and of the optimal wavelength $\left(\lambda_{\mathrm{opt}}\right)$ as given by system specifications. The curve $Z(\alpha)$ is represented in Fig. 8 .

$2|\partial \lambda|$ and $\partial W$ are proportional to the square of the normalized beam waist $\left(d_{0} / D\right) \in[0,1]$. This term is related to the degree of overlapping of the self-images, and can therefore not be increased to values much higher than $1 / 2$ in practical applications. The optical bandwidth of MMI couplers is inversely proportional to the number of input and output channels $N$. This behavior can be a strong limitation for using a large value of $N$. The fabrication tolerances are independent of $N$ and proportional to the channel spacing $D$.

\section{B. Examples and Comparison with Mode Analysis (MA)}

We consider the InP/InGaAsP waveguide structure of Fig. 3. With this structure, polarization-independent switches have been realized [5]. Optical bandwidth as well as fabrication tolerance measurements of MMI couplers using this waveguide structure will be presented.

We first consider restricted interference MMI couplers described by (6). The inputs and outputs of width $w=3 \mu \mathrm{m}$ are positioned at $W / 3$ and $2 W / 3$, in order not to excite the modes $2,5,8, \cdots$ For a geometrical width of $W=18 \mu \mathrm{m}$, the equivalent width is $W_{\text {eq }}=19.2 \mu \mathrm{m}$ and $19.0 \mu \mathrm{m}$ for TE and TM, respectively; this is due to the lateral penetration of the evanescent fields at the side of the MMI section. The parameters $a, n$, and $d_{0}$ have the values $a=3, n=3.3$, $d_{0}=3.4 \mu \mathrm{m}$. The length is $L=L_{c} / 2=530 \mu \mathrm{m}$ for the $3 \mathrm{~dB}$ MMI coupler (two images of equal intensity at the outputs) and $L=L_{c}=1060 \mu \mathrm{m}$ for the cross-MMI coupler (one image at the cross-channel output). In Fig. 4, the optical bandwidth and the fabrication tolerances described by (6) are compared with the results obtained by the more sophisticated mode Analysis (MA) method [12] for the TE polarization. With the MA, no strong guiding and paraxial approximations are used, and effects like image broadening can be described. This leads to a nonzero excess loss at the optimum. Except for these losses, the bandwidth and fabrication tolerances calculated with both methods are very similar.

Then we analyze the case of a general $N \times N$ MMI coupler with equidistant inputs/outputs corresponding to (7). The structure of Fig. 3 is used with a constant channel spacing $D$. In Fig. 5(a) the inversely proportional dependency of the optical bandwidth on $N$ is illustrated. The optical bandwidth of MMI couplers becomes a limiting parameter for values of $N$ greater than 8 . The dependency of the fabrication tolerances on $D$ is shown in Fig. 5(b). The value of $D$ should remain somewhat greater than $d_{0}$ in order to assure well-separated output images. From (7), we infer that scaling down both $D$ and $d_{0}$, keeping the ratio $d_{0} / D$ constant, will linearly tighten the fabrication tolerances, but the MMI coupler length $L$ will quadratically decrease (2).

\section{EXPERIMENT}

MMI couplers with the same waveguide structure (Fig. 3) as for the theoretical calculations of Fig. 4 have been produced on InP/InGaAsP material. These devices are fully compatible with an electrooptic phase shifter and can be integrated in polarizedindependent four-port Mach-Zehnder optical switches [5].

A number of $3 \mathrm{~dB}$ MMI couplers, as well as cross-MMI couplers with restricted interference have been integrated, with $W$ ranging from 17.5 to $18.5 \mu \mathrm{m}$ in steps of $0.25 \mu \mathrm{m}$. The $3 \mathrm{~dB}$ MMI couplers have a length $L=L_{c} / 2=530 \mu \mathrm{m}$ and divide the intensity of each input port equally into both output channels. The cross-MMI couplers have a length $L=L_{c}=$ $1060 \mu \mathrm{m}$ and connect each input to the corresponding cross output. The input and output waveguides were placed at $1 / 3$ and $2 / 3$ of the equivalent MMI coupler width in order to obtain a restricted interference mechanism by not exciting the modes $2,5,8, \cdots[12]$. Curved access waveguides with offsets were applied with a radius of $2 \mathrm{~mm}$ and a width of $3 \mu \mathrm{m}$.

A thick InP top and weakly doped layers adjacent to the quaternary InGaAsP guiding layer keep the absorption losses 


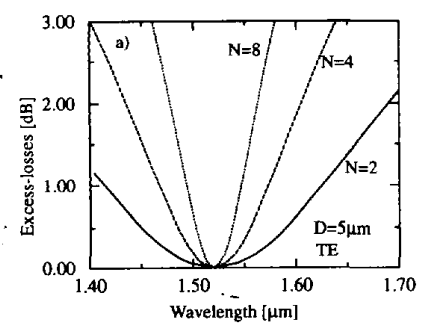

(a)

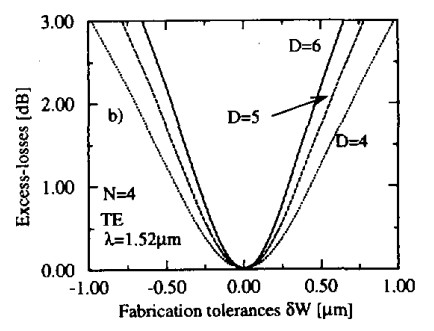

(b)

Fig. 5. (a) Optical bandwidth of general $N \times N$ MMI couplers with equidistant inputs and outputs. The curves are calculated with (7). $D=5 \mu \mathrm{m}$. (b) Fabrication tolerances of general $N \times N$ MMI couplers with equidistant inputs/outputs. The curves are calculated with (7). $N=4, \lambda=1.52$ $\mu \mathrm{m} . M=1, a=1, d_{0}=3.4 \mu \mathrm{m}, n=3.3$, TE polarization.

low. The layers were grown by metal-organic vapor phase epitaxy (MOVPE) on an $\mathbf{n}^{+}$InP substrate. The waveguides were patterns by magnetron-enhanced $\mathrm{CH}_{4} / \mathrm{H}_{2}$ reactive ion etching.

For the measurements, the devices were placed between $40 \times$ IR anti-reflection-coated microscope objectives. The light of a wavelength-tunable external grating semiconductor laser was launched into each MMI coupler input and recorded from both outputs by a Ge photodiode. Measurements with TE and TM polarized light were successively performed.

We define the excess losses $\alpha$ and the unbalance (UB) due to wavelength and width variations by

$$
\begin{gathered}
\alpha \equiv-10 \cdot \log _{10}\left(\frac{\left(P_{\text {bar }}+P_{\text {cross }}\right)}{\left(P_{\text {bar }}+P_{\text {cross }}\right)_{\text {opt }}}\right) \text { and } \\
\mathrm{UB} \equiv 10 \cdot \log _{10}\left(\frac{P_{\text {bar }}}{P_{\text {cross }}}\right) .
\end{gathered}
$$

The value $\left(P_{\text {bar }}+P_{\text {cross }}\right)_{\text {opt }}$ corresponds to the sum of the two output intensities at the optimal wavelength and MMI coupler width. We first measured MMI couplers with a geometrical width $W=18 \mu \mathrm{m}$ at various wavelengths. Then MMI couplers with different widths $W$ were measured at fixed wavelength $\lambda=1.52 \mu \mathrm{m}$. The measurements are compared with the analytic approximations of (6) and Fig. 4.

The measurements obtained with $3 \mathrm{~dB}$ MMI couplers of length $L=530 \mu \mathrm{m}$ are shown in Fig. 6. The results for both polarizations overlap almost completely. The couplers are, to a good approximation, polarization independent. The unbalance remains close to $0 \mathrm{~dB}$ and is, on the entire measurement region, never greater than $1 \mathrm{~dB}$. The unbalance measurements compare well with the predictions of the MA (dashed line).

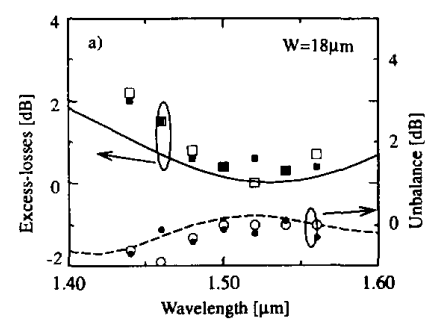

(a)

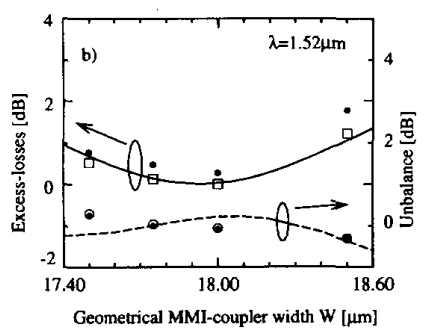

(b)

Fig. 6. (a) Measured optical bandwidth of $3 \mathrm{~dB}$ MMI couplers as in Fig 4. (b) Measured fabrication tolerances of $3 \mathrm{~dB}$ MMI couplers as in Fig. 4. Circles $=$ unbalance measurements, squares $=$ excess loss measurements. Open symbols $=$ TE polarization, solid symbols $=\mathrm{TM}$ polarization. Solid line $=$ analytic approximation (6) for the TE excess losses. Dashed line = MA simulation of the TE unbalance.

The excess losses increase up to $2 \mathrm{~dB}$ at the sides of the measurement region and limit the optical bandwidth and fabrication tolerances. The experimental excess losses are in good agreement with the analytical approximation of (6), especially for the fabrication tolerances. The small discrepancy of the predicted optical bandwidth in Fig. 6(a) at short wavelength is probably due to the fact that, in our approximation, the index dispersion and the increase of the material absorption in this wavelength region are neglected. With a limit of $1 \mathrm{~dB}$ excess losses, an optical bandwidth of more than $100 \mathrm{~nm}$ and fabrication tolerances of about $\pm 0.5 \mu \mathrm{m}$ have been predicted and measured for these $3 \mathrm{~dB}$ MMI couplers.

Measurement results for cross couplers with length $L=$ $1060 \mu \mathrm{m}$ are presented in Fig. 7. Again, a polarizationindependent behavior of the MMI couplers is obtained, due to the lateral strong guided MMI section. The experimental data fit well with the analytical predictions of the previous section. Compared with the $3 \mathrm{~dB}$ MMI couplers of Fig. 6, the optical bandwidth and the fabrication tolerances are reduced. This corresponds to the reduction of $a N / M$ in (6). For $1 \mathrm{~dB}$ excess losses, the measured and predicted optical bandwidth and fabrication tolerances are about $80 \mathrm{~nm}$ and $\sim \pm 0.25$ $\mu \mathrm{m}$. We also measured the cross talk defined exactly like the unbalance, but for the cross-MMI couplers. It remains smaller than $-20 \mathrm{~dB}$, except for the case of $\lambda<1.45 \mu \mathrm{m}$ and $W=18$ $\mu \mathrm{m}$ where it increases slightly to around $-15 \mathrm{~dB}$. As expected, the bandwidth and the tolerances are limited by the excess losses.

\section{CONCLUSIONS}

The optical bandwidth and the fabrication tolerances of MMI couplers have been analytically studied. We derived 


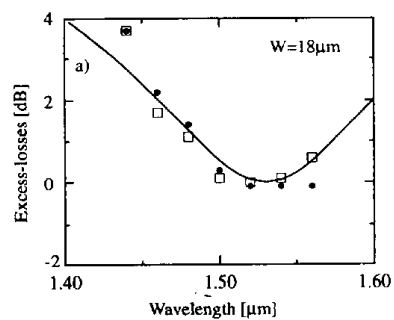

(a)

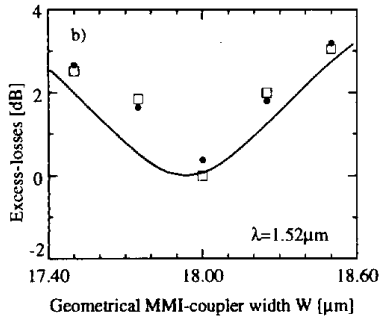

(b)

Fig. 7. (a) Measured optical bandwidth of cross-MMI couplers as in Fig 4. (b) Measured fabrication tolerances of $3 \mathrm{~dB}$ MMI couplers as in Fig. 4 Circles $=$ unbalance measurements, squares $=$ excess loss measurements Open symbols $=\mathrm{TE}$ polarization, solid symbols $=\mathrm{TM}$ polarization. Solid line $=$ analytic approximation (6) for the TE excess losses.

simple expressions that relate these two basic properties of MMI couplers to the main design parameters. The optical bandwidth is shown to be inversely proportional to the number of input and output waveguides $N$, while the tolerances are independent of $N$ but are proportional to the waveguide separation $D$. Both $2|\partial \lambda|$ and $\partial W$ are proportional to the square of the normalized beam waist $\left(d_{0} / D\right)$, itself directly related to the degree of overlapping of the self-images.

We successfully compare these analytic expressions with more sophisticated mode analysis simulations and to measurement results obtained using an $\mathrm{InP} / \mathrm{InGaAsP}$ waveguide structure compatible for switch integration. We realized and measured $3 \mathrm{~dB}$ MMI couplers as well as cross-MMI couplers based on restricted mode interference. For $1 \mathrm{~dB}$ excess losses, optical bandwidths of more than $100 \mathrm{~nm}$ and of about $80 \mathrm{~nm}$ have been found for $3 \mathrm{~dB}$ and cross couplers, respectively, while the fabrication tolerances are \pm 0.5 and $\pm 0.25 \mu \mathrm{m}$, respectively.

\section{APPENDIX}

In this Appendix, we derive the relation between the length variation $\delta L$ and the excess losses $\alpha$. We consider a Gaussianbeam propagating in the $z$, diverging in the $x$, and maintaining its profile in the $y$ direction. Its focus is at $z=0$ and its Gaussian waist is $d_{0}$. The amplitude can be written as

$$
u(z, x)=\left(\frac{8}{\pi d^{2}}\right)^{1 / 4} \cdot e^{-(2 x / d)^{2}} \cdot e^{i\left(\pi n x^{2} / \lambda R\right)} \cdot e^{i \varphi(z)}
$$

with $\varphi(z)$ a phase term independent of $x$. The parameters $R$

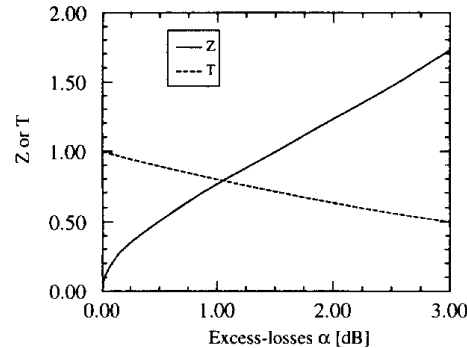

Fig. 8. Transmission $T$ and values of the parameter $Z$ as a function of the excess losses $\alpha$.

and $d$ are defined as

$$
\begin{gathered}
R(z) \equiv z \cdot\left(1+\left(\frac{1}{2 \Sigma}\right)^{2}\right) \text { and } \\
d^{2}(z) \equiv d_{0}^{2}+(2 \Sigma)^{2} \quad \text { with } \quad \Sigma \equiv \frac{2 \lambda z}{\pi n d_{0}^{2}} .
\end{gathered}
$$

The losses can be calculated by overlapping this Gaussian beam focused at $z=0$, with the same Gaussian beam but focused at $z=\delta L$. We get, after complex integration,

$$
\begin{aligned}
\langle u(0, x) \mid u(\partial L, x)\rangle & \equiv \int_{-\infty}^{\infty} u(0, x) u^{*}(\partial L, x) d x \\
& =\frac{\sqrt{2} \cdot \sqrt[4]{1+4 Z^{2}}}{\sqrt{\left(2+4 Z^{2}\right)+i(2 Z)}} \cdot e^{-i \varphi(z)}
\end{aligned}
$$

with the definition $Z \equiv(2 \lambda \partial L) /\left(\pi n d_{0}^{2}\right)$.

The excess losses $\alpha$ are related to the transmission $T$ by $\alpha \equiv-10 \cdot \log _{10}(T)$ with

$$
T \equiv|\langle u(0, x) \mid u(\partial L, x)\rangle|^{2}=\frac{\sqrt{1+4 Z^{2}}}{\sqrt{1+5 Z^{2}+4 Z^{4}}} .
$$

Solving this equation in $Z$ leads to (5) for $T \in[0,1]$. The relationship among $\alpha, Z$, and $T$ can be found in Fig. 8 .

\section{REFERENCES}

[1] O. Bryngdahl, "Image formation using self-imaging techniques," J. Opt. Soc. Amer., vol. 63, no. 4, pp. 416-418, 1973.

[2] R. Ulrich and T. Kamiya, "Resolution of self-images in planar optical waveguides," J. Opt. Soc. Amer., vol. 68, no. 5, pp. 583-592, 1978.

[3] J. M. Heaton, R. M. Jenkins, D. R. Wight, J. T. Parker, J. C. H. Birbeck, and K. P. Hilton, "Novel 1-to- $N$ way integrated optical beam splitters using symmetric mode mixing in GaAs/AlGaAs multimode waveguides," Appl. Phys. Lett., vol. 61, no. 15, pp. 1754-1756, 1992.

[4] A Ferreras, F. Rodriguez, E. Gomez-Salas, J. de Miguel, and F. Hernandez-Gil, "Design and fabrication of InP/nGaAsP multimode power splitter" in Proc. IPRC'93, paper IME4-1, pp. 151-154.

[5] M. Bachmann, M. K. Smit, P. A. Besse, E. Gini, H. Melchior, and L. B. Soldano, "Polarization-insensitive low-voltage optical waveguide switch using InGaAsP/InP four-port Mach-Zehnder interferometer," in Tech. Dig. OFC/IOOC'93, San Jose, CA, Feb. 1993, pp. 32-33.

[6] J. E. Zucher, K. L. Jones, T. H. Chiu, B. Tell, and K. Brown-Goebeler, "Strained quantum wells for polarization-independent electrooptic waveguide switches," J. Lightwave Technol, vol. 10, pp. 1926-1930, Dec. 1992

[7] J. Deri, E. C. M. Pennings, A. Scherer, A. S. Gozdz, C. Caneau, N. C. Andreadakis, V. Shah, L. Curtis, R. J. Hawkins, J. B. D. Soole, and J.I. Song, "Ultracompact monolithic integration of balanced, polarization diversity photodetectors for coherent lightwave receivers," IEEE Photon. Technol. Lett., vol. 4, pp. $1238-1240$, Nov. 1992. 
[8] E. Pennings, R. Deri, R. Bhat, T. Hayes, and N. Andreadakis, "Ultracompact integrated all-passive optical $90^{\circ}$ hybrid using self-imaging," in Proc. ECOG'92, Berlin, paper We B10.4, pp. 461-464.

[9] M. van Stralen, R. van Roijen, E. Pennings, J. van der Heijden, T. van Dongen, and B. Verbeek, "Design and fabrication of integrated InGaAsP ring lasers with MMI-couplers," in Proc. ECIO'93, Neuchatel, paper 2-24.

[10] L. Soldano, F. Veerman, M. Smit, B. Verbeek, A. Dubost, and E. Pennings, "Planar monomode optical couplers based on multimode interference effects," J. Lightwave Technol., vol. 10, pp. 1843-1849, Dec. 1992

[11] P. Besse, M. Bachmann, and H. Melchior, "Phase relations in multimode interference couplers and their application to generalized integrated Mach-Zehnder optical switches," in Proc. ECIO'93, Neuchatel, paper 2-22.

[12] L. Soldano, M. Bachmann, P. Besse, M. Smit, and H. Melchior, "Large optical bandwidth of In GaSaP/InP multi-mode interference 3-dB couplers," in Proc. ECIO'93, Neuchatel, paper 14-10.

[13] L. Soldano, F. Veerman, M. Smit, and B. Verbeek, "High-performance monomode planar couplers using a short multi-mode interference section," in Proc. ECOC'91, Paris, paper TuB5-2, pp. 225-228.

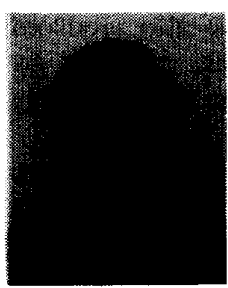

Pierre A. Besse was born in Sion, Switzerland, in 1961. He received the diploma in physics and the doctorate on semiconductor optical amplifiers from the Swiss Federal Institute of Technology, ETH Zurich, in 1986 and 1992 , respectively.

In 1986 he joined the Group of Micro- and Optoelectronics of the Institute of Quantum Electronic at ETH Zurich, where he is engaged in research on optical telecommunication science. His major fields of interest are theories, modeling, characterization techniques, and fabrication of compound semiconductor devices such as polarization-independent optical switches and modulators, passive optical components like star couplers, multimode interference couplers, and wavelength demultiplexers on III-V materials, as well as polarization-independent traveling-wave semiconductor optical amplifiers and external cavity tunable laser sources.

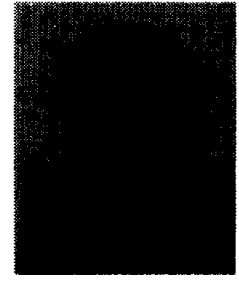

Maurus Bachmann was born in Grabs, Switzerland, on June 9, 1961. He studied at the Swiss Federal Institute of Technology, ETH Zurich, where he received the diploma in physics in 1986.

He is currently working towards the Ph.D. degree at the Swiss Federal Institute of Technology, Zurich. His present work deals with theories, design, fabrication, and characterization techniques of InGaAsP/InP-based integrated optical components for telecommunications. His major fields of interest are polarization-independent optical switches and modulators, as well as passive integrated optical components such as multimode interference couplers.

H. Melchior, photograph and biography not available at the time of publication.

L. B. Soldano, photograph and biography not available at the time of publication.

M. K. Smit, photograph and biography not available at the time of publication. 